\title{
Complex World: um jogo para auxiliar o aprendizado de inglês como segunda língua utilizando a abordagem pedagógica do Pensamento Complexo
}

\author{
Renato Cristiano Montanher ${ }^{1}$, Igor Calebe Zadi ${ }^{1}$, Ana Maria Monteiro ${ }^{2}$ \\ ${ }^{1}$ Grupo de Informática e Tecnologia em Educação e Sociedade - GITES \\ Instituto Federal de São Paulo (IFSP) - São Paulo - SP - Brazil \\ ${ }^{2}$ Centro Universitário Campo Limpo Paulista (UNIFACCAMP) \\ \{renato.montanher,igor.zadi\}@ifsp.edu.br, anammont.alu@gmail.com
}

\begin{abstract}
This paper introduces Complex World, a digital RPG created to assist in teaching and learning English as a second language. Complex World uses a pedagogical approach based on Complex Thinking and has a chatbot as an interlocutor. High school and higher education students used Complex World and the results of this experiment show that the developed RPG had a positive impact on motivation, on the user experience, on the questions of dialogue and learning. It was possible to verify that Complex World proved to be a viable and promising option to assist in learning a second language.
\end{abstract}

Resumo. Este artigo apresenta Complex World, um RPG digital criado para auxiliar no ensino-aprendizado de Inglês como segunda língua. Complex World utiliza uma abordagem pedagógica baseada no Pensamento Complexo e possui um chatbot como interlocutor. Estudantes do ensino médio e superior utilizaram Complex World e os resultados desse experimento apontam que o RPG desenvolvido impactou positivamente na motivação, na experiência do usuário, nos quesitos de interlocução e aprendizagem. Foi possível verificar que Complex World se mostrou uma opção viável para auxiliar no ensinoaprendizagem de uma segunda língua.

\section{Introdução}

O aprendizado de uma língua estrangeira é um tema que ganha cada vez mais importância e destaque. Muitos pesquisadores da área buscam-encontrar formas de ensino mais eficazes e motivadoras para os estudantes. A tecnologia aliada a tais formas de ensino pode contribuir para tornar o ensino de línguas mais eficaz, bem como atingir um maior número de pessoas, tornando o processo de ensino/aprendizado mais acessível e motivador.

Uma das possíveis maneiras de alcançar tal motivação é através de jogos. $\mathrm{O}$ ato de jogar, além de proporcionar prazer, é um meio do sujeito desenvolver habilidades de raciocínio e cognição, estimulando a atenção e a memória [Furió et al. 2013]. Os jogos atuam como um meio capaz de motivar os indivíduos, apresentando-se como uma alternativa eficiente no processo de geração de conhecimento [Busarello 2016].

Uma funcionalidade desejada em um jogo para o ensino de uma língua estrangeira é um interlocutor para que o usuário possa praticar. A possibilidade de um interlocutor 
inteligente faz parte das pesquisas relacionadas com o aprendizado de idiomas mediado por computador, em especial do aprendizado de idiomas mediados por ferramentas inteligentes [Do Amaral 2007; Taalas et al. 2018].

O ensino de uma segunda língua para a nova geração de estudantes tem seus próprios desafios, levando em consideração que as pessoas dessa geração podem ser chamadas de nativos digitais [Prensky 2018], pois são pessoas que nasceram e cresceram em meio aos mais variados tipos de recursos tecnológicos, como celulares, videogames e computadores. Com isso, elas desenvolveram habilidades diferentes da geração predecessora, também conhecida como imigrantes digitais. Dentre as características mais marcantes presentes nos nativos digitais estão a capacidade de desenvolver o conhecimento utilizando múltiplas fontes, de forma aleatória, o imediatismo, a necessidade da sensação de progressão e um amplo consumo de aplicativos, incluindo jogos digitais. Essas características se tornam mais problemáticas quando essa geração, ao ir à escola, se depara com professores imigrantes digitais que utilizam metodologias não aderentes à nova geração, visto que em muitas escolas o conhecimento é desenvolvido de forma linear e os alunos têm pouca autonomia no processo ensinoaprendizagem.

Entendendo essas características, este artigo apresenta o protótipo de jogo denominado Complex World, que considerou na sua construção conceitos referentes a jogos digitais, design de conversação utilizando chatbots e conceitos de uma metodologia de ensino denominada Pensamento Complexo [Morin 2015] para tentar atender as necessidade de aprendizagem de nativos digitais para uma segunda língua. Também são apresentados os resultados de um experimento realizado para verificar o impacto do seu uso quanto à motivação e a vontade de se comunicar dos alunos ao interagirem com o protótipo e aos aspectos de aprendizagem.

\section{Trabalhos relacionados}

Os trabalhos foram oriundos de artigos publicados entre os anos de 2010 e 2020 nos repositórios IEEEXplore, SpringLink, ACM, ScienceDirect e Google Scholar. As palavras chaves utilizadas na busca foram: "foreign language learning", "games" e "chatbot education". Foram incluídos artigos que abordassem o uso de jogos e/ou chatbots na área de educação, relacionados ao ensino de uma língua estrangeira e que eventualmente utilizem alguma abordagem pedagógica no ensino dessa língua.

Entre os trabalhos que fazem uso de uma abordagem pedagógica, pode-se mencionar o artigo "Mobile Support for Learning of Foreign Languages" [Behúnová et al. 2016] que apresenta um protótipo desenvolvido utilizando o método Callan com ênfase na expressão oral através de diálogos com questões e respostas rápidas. No trabalho "MicroMandarin: Mobile Language Learning in Context" [Edge et al. 2011] foi criado um protótipo baseado em microaprendizado contextual, termo vindo da psicologia cognitiva que faz uso de repetição espaçada de conteúdo, conhecimento baseado em atividade, contexto e cultura.

No artigo "An evaluative study of a mobile application for middle school students struggling with English vocabulary learning" [Hao et al. 2019] foi desenvolvido um protótipo de um jogo baseado na Teoria de Aprendizagem Cognitiva que utiliza missões e resolução de quebra-cabeças, tudo guiado por narrativas para o jogador viver situações autênticas. $\mathrm{O}$ artigo "Design and Implementation of a 3D Multi-User Virtual World for 
Language Learning" [Ibáñez et al. 2016] relata uma pesquisa que utiliza dois aspectos da abordagem de ensino construtivista, o aprendizado situado e o aprendizado cooperativo/colaborativo. Um protótipo foi implementado e foram feitas avaliações de motivação, da imersão e sobre a experiência do usuário. Já o trabalho "Mobile Navigator for Learning Foreign Languages" [Kalita et al. 2015] apresenta um protótipo que utiliza como abordagem pedagógica a metodologia orientada a etnia, na qual o conhecimento referente à uma nova língua é construído levando em conta os conhecimentos já adquiridos no contexto da cultura do aluno.

No trabalho "Motivation, students' needs and learning outcomes: a hybrid game-based app for enhanced language learning" [Berns et al. 2016] foi desenvolvido um jogo que apresenta o conteúdo utilizando a estratégia de treino e repetição focado em gramática e um jogo de detetive no qual os usuários recebem dicas através de um chat para descobrir quem é o assassino. No artigo "O aprendizado de língua inglesa em dispositivos móveis através da aplicação de um sistema tutor inteligente", [Silva e Teles 2011] descrevem uma aplicação para dispositivos móveis e analisam a interação do usuário com a aplicação e seus efeitos na aprendizagem da língua.

Todos os trabalhos mencionados anteriormente relatam que foram obtidos bons resultados no processo de ensino e aprendizagem. Nenhum dos trabalhos achados fazem uso do Pensamento Complexo como metodologia de ensino.

Com relação ao uso de chatbots, pode-se mencionar o artigo "Chatbot learning partners: Connecting learning experiences, interest and competence" [Fryer et al. 2019] no qual são comparadas as interações entre alunos que utilizam chatbots e interlocutores humanos. $\mathrm{O}$ artigo relata aspectos positivos do uso de tal tecnologia no ensino de línguas estrangeiras. Outra pesquisa que também indica uma melhora do engajamento quando se utiliza a tecnologia dos chatbots é o artigo "BookBuddy: Turning Digital Materials Into Interactive Foreign Language Lessons Through a Voice Chatbot" [Ruan et al. 2019]. Nessa pesquisa foram registradas as emoções de crianças que interagiram com um chatbot desenvolvido como um assistente virtual.

$\mathrm{O}$ artigo "Learning to Read/Type a Second Language in a Chatbot Enhanced Environment" [De Gasperis and Florio 2012] compara duas concepções de projeto aplicados no desenvolvimento de chatbots: um voltado para a leitura e digitação, onde o de um conjunto específico de palavras, e outro chatbot para perguntas elaboradas a partir de um texto fornecido pelo professor. A pesquisa conclui possíveis tipos de avaliações para cada uma das abordagens. No artigo "Application of Mobile Learning System in Phonetics Teaching" [Jing 2017] investiga-se a eficácia do uso de um sistema móvel com chatbot para ensinar fonética. E relata que o uso do aplicativo melhorou a consciência fonética dos alunos e o nível fonético. Ayedoun e seus colaboradores (2015) em " $A$ Conversational Agent to Encourage Willingness to Communicate in the Context of English as a Foreign Language" propõem o desenvolvimento e avaliação de um chatbot que utiliza o modelo "vontade para se comunicar". O chatbot fornece aos usuários vários contextos diários de conversação com o intuito de tornar as conversas mais realistas e eficientes. A pesquisa indica que o uso frequente do sistema pode aumentar a autoestima dos alunos dando-lhes confiança e desejo de se comunicar em inglês.

A maioria dos trabalhos utilizam situações de treinamento do cotidiano do aluno, intenção também presente no trabalho aqui apresentado. 


\section{Fundamentos teóricos}

A seguir serão apresentados os conceitos fundamentais referentes à metodologia de ensino utilizada, jogos digitais e chatbots. Esses conceitos nortearam a definição e desenvolvimento do Complex World.

\subsection{Metodologia de ensino}

Considerando aspectos observados nos trabalhos relacionados, a escolha de uma metodologia de ensino se apresenta como uma possibilidade interessante para um ganho no processo de construção do conhecimento. Nesse sentido, a metodologia escolhida foi a Teoria do Pensamento Complexo que se origina nos estudos do filósofo Edgar Morin [Morin 2015]. Para entender a ideia do Pensamento Complexo deve-se primeiramente compreender que o sentido de complexidade se remete à etimologia de complexus, que faz referência a um conjunto entrelaçado de constituintes heterogêneas inseparavelmente associadas. E tais elementos são atualmente estudados em diversas áreas do conhecimento, principalmente na educação.

Tal paradigma vem sendo utilizado tanto em novos projetos educacionais [Freire 2013] quanto no ensino de línguas [Leffa 2016]. A língua por si só pode ser considerada um sistema complexo, pois os elementos que a compõem, os sons, as palavras, as frases e os textos não atuam de maneira isolada, mas interagem com outros sistemas, seja internamente dentro da própria língua, ou externamente com elementos sociais, contextuais ou simplesmente extralinguísticos.

Existem três princípios que ajudam no entendimento do Pensamento Complexo: o princípio dialógico, o princípio de recursão organizacional e o princípio hologramático.

De acordo com Morin (2015), o todo se constitui em algo mais do que a mera soma das partes, o princípio dialógico permite manter a dualidade no seio da unidade associando dois termos simultaneamente complementares e antagônicos. Por exemplo, o entendimento do ruim é mais significativo quando se entende o que é bom. Esses termos antagônicos se complementam e ajudam no entendimento um do outro.

A recursão organizacional, segundo Morin (2015), pode ser entendida como um processo de influência bidirecional, onde o produtor influencia seu produto e vice-versa, trocando assim os papéis no processo. Esse conceito estrutura uma ideia de ruptura com relação à ideia linear de causa/efeito, de produto/produtor, de estrutura/superestrutura, uma vez que tudo que é produzido volta-se sobre o que o produz num ciclo auto constituinte, auto organizador e autoprodutor.

Por fim, o princípio hologramático, como sugere Morin (2015), considera que em um sistema ou um mundo complexo, não apenas a parte encontra-se no todo, mas o todo encontra-se na parte. Não apenas o indivíduo existe em uma sociedade, mas a sociedade existe em seu interior, uma vez que desde seu nascimento a sociedade inseriu nele a linguagem, a cultura, suas proibições e suas normas.

A discussão sobre Pensamento Complexo vai muito mais além do que a explanação desses princípios, contudo, essas definições foram consideradas no desenvolvimento do jogo Complex World, no qual é criada uma estrutura social que promove o desenvolvimento de diálogos que se complementam e auxiliam no entendimento um do outro e, dessa forma, ajudam na construção do conhecimento do aluno. 


\subsection{Jogos Digitais}

Segundo Roggers (2010) um jogo (do inglês, game) é em uma atividade que tem pelo menos um jogador, tem regras e tem condições de vitória. Caso esse jogo seja exibido numa tela, se torna um vídeo game. Vídeo games tem a capacidade de prender seus jogadores por horas, devido ao extenso uso de elementos que aumentam o foco e a motivação nas atividades dentro do mundo virtual. Além disso, para Collantes (2013), os jogos são capazes de promover contextos lúdicos e ficcionais na forma de narrativas que favorecem o processo de geração e relação com o conhecimento. Essa forma de construir o conhecimento também é defendida pelo Pensamento Complexo, que entende que toda sensação, que pode ser desenvolvida ao longo de uma narrativa, ajuda na construção do conhecimento. Nesse mesmo sentido, Steiner e Tomkins (2010) entendem que a narrativa é uma forma de abordar o mundo que possibilita que os mais variados temas possam ser vividos de forma emocional, com isso, é possível construir jogos com os mais variados temas e assim construir conhecimento dos mais variados assuntos.

Existem vários gêneros de jogos como ação (action), atirador (shooter), aventura (adventure), construção (construction), quebra-cabeça (puzzles) etc. Dentre eles, existe o chamado RPG (do inglês, Role - Playing Game), um subgênero dos jogos de aventura [Rogers 2015], que tem como características principais o foco em resolução de enigmas, a coleta de itens e o gerenciamento de inventários. Além disso, esse estilo de jogos baseiase fortemente em narrativas. O gênero RPG em jogos digitais nasceu do RPG de mesa, que é um jogo não digital em que os jogadores devem interpretar personagens e viver aventuras de acordo com o que o mestre do jogo (narrador da história) apresenta para eles. Essa característica de construção narrativas presente em jogos RPG é o que torna esse gênero interessante para o projeto em consonância com a metodologia de ensino escolhida. A utilização deste tipo de jogo abre a possibilidade de construção de diversos fluxos narrativos, nos quais é possível utilizar diálogos presentes no dia a dia e que mostrem, de forma contextualizada, o uso real de vocábulos de uma segunda língua.

\subsection{Chatbot e WTC}

Um chatbot, é um software que tem por objetivo replicar uma conversa humana simulando as interações de uma pessoa. Atwell (1999) observava que este tipo de software poderia oferecer para os alunos a oportunidade de realizarem práticas de conversação além da sala de aula. Levando em consideração que o uso de chatbots realizando atendimento digital cresceu a partir de 2020 devido à pandemia de COVID-19 e os chatbots estão presentes no dia a dia, especialmente dos nativos digitais, seu uso pode ser eficaz para o aprendizado de uma língua estrangeira.

Segundo MacIntyre et al. (1998) um fator fundamental para o uso de uma língua estrangeira e a vontade de se comunicar ( Willingness To Communicate - WTC) que pode ser definida como a prontidão para participar de um diálogo em um determinado momento com uma pessoa ou várias pessoas utilizando uma língua estrangeira. Segundo esses autores, o uso da língua e a comunicação efetiva em uma língua estrangeira podem ser vistos como o resultado de um complexo sistema com diversas variáveis interrelacionadas. O comportamento de comunicação, em um sentido amplo, inclui atividades como falar com a turma da classe, ler jornais, assistir televisão ou até mesmo o seu uso no trabalho. O material instrutivo ou o curso deve ter como objetivo gerar nos estudantes a vontade de se comunicar [MacIntyre et al. 1998]. 
A vontade de se comunicar baseia-se na autoconfiança desenvolvida no aprendizado anterior da língua e na falta de ansiedade combinada com um nível suficiente de competência comunicativa, decorrente de uma série de experiências com a língua estrangeira razoavelmente agradáveis [MacIntyre et al. 1998]. Uma outra variável importante para não perder a vontade de continuar se comunicando é a empatia com o interlocutor e o fato de ter vivenciado situações análogas na língua materna.

\section{Complex World}

Complex World foi idealizado para tentar atender o máximo possível das necessidades do nativo digital. O protótipo tem como público-alvo estudantes que se enquadram no nível A1 e B1 de compreensão de uma língua estrangeira ${ }^{1}$.

Complex World é um jogo do tipo RPG, concebido seguindo princípios do Pensamento Complexo, que retrata a história do personagem Herrera, um jovem viajante que acorda em uma casa misteriosa no vilarejo conhecido como Woodville. Ao comunicar-se com as pessoas deste local incomum, Herrera descobre que todos ali falam em inglês, com exceção apenas de um sábio ancião chamado Joaquim, que domina não só o inglês, mas também o português, a língua materna do protagonista Herrera. Ao decorrer do jogo, Herrera precisa interagir com os habitantes de Woodville, superar desafios como falar inglês e descobrir uma forma de retornar para sua casa.

O jogo conta com dois ambientes, uma área residencial e uma área central onde existem algumas lojas, um banco. Nesses ambientes, apresentados na Figura 1, se desenrolam as missões que o jogador deverá cumprir.

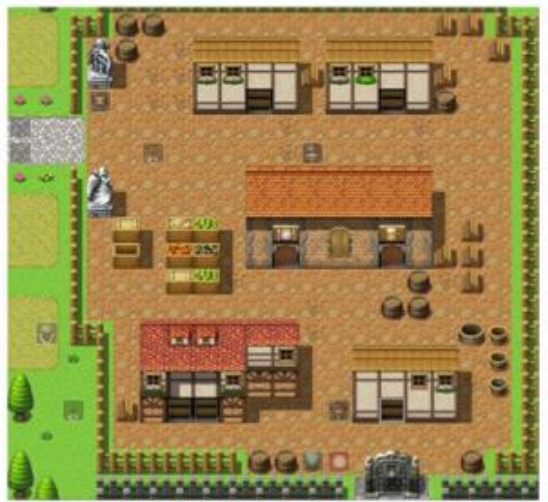

a) área Residencial

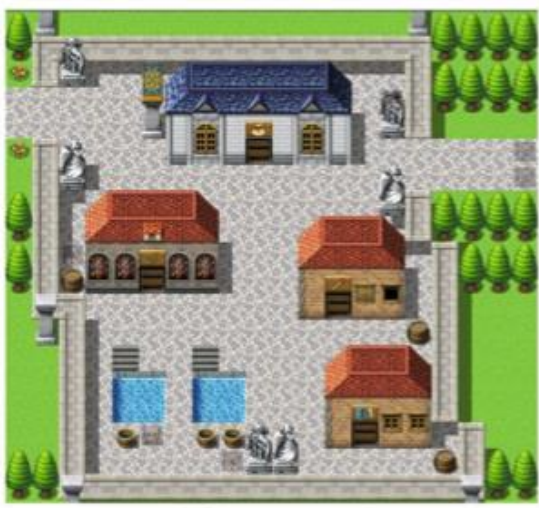

b) área Central

Figura 1 - Ambientes do jogo

Outro ponto de destaque do jogo é a integração com um chatbot que se apresenta no jogo como um personagem, o mago Billy. No que diz respeito à narrativa, para integrar o jogo com o chatbot, o jogador precisa de interações constantes com o chatbot para obter informações necessárias para poder realizar as missões. A apresentação do chatbot no jogo pode ser vista na Figura 2.

Para desenvolver o protótipo do jogo foi escolhido o motor RPG Maker, pelo fato de ter foco no gênero RPG e fornece muitos recursos já implementados para jogos dessa

\footnotetext{
${ }^{1}$ De acordo com o Common European Framework of Reference for Languages (CEFR) disponível em: https://www.coe.int/en/web/common-european-framework-reference-languages/level-descriptions
} 
modalidade, recursos tais como gerenciamento de inventário, controle de progresso, sistemas de batalhas etc. Em virtude dessas características, esse motor mostrou-se mais produtivo por permitir fazer uma rápida prototipação, tornando possível assim investir menos tempo em programação de componentes e mais na criação de conteúdo para o projeto. Para a implementação do chatbot foi escolhido Dialogflow por fornecer uma interface que facilita a criação e testes do bot e possui integração web nativa, o que facilita a integração com o RPG.

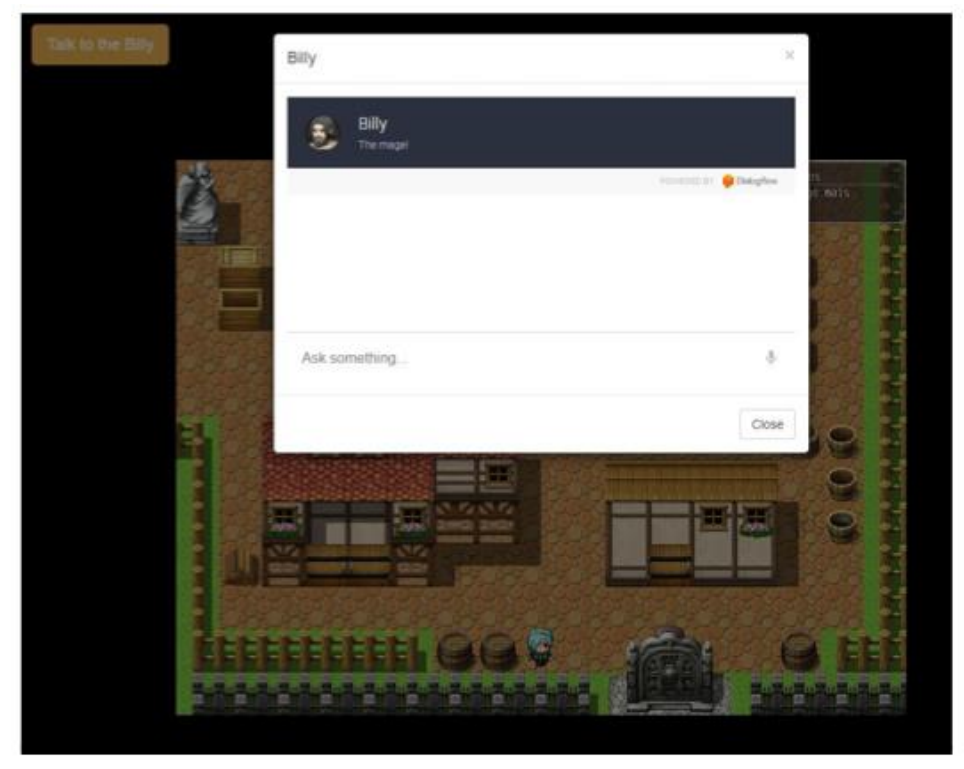

Figura 2 - Apresentação do chatbot no jogo

\section{5. $O$ experimento}

O modelo de avaliação utilizado foi o modelo proposto por Savi (2010) para a avaliação de jogos educacionais. Tal modelo é composto de conceitos baseados em teorias da área de design instrucional e educação como o modelo ARCS, a taxonomia de Bloom, o modelo de Kirkpatrick, e em uma compilação de estudos recentes da área de experiência do usuário em jogos.

Para a avaliação do chatbot foram utilizados os conceitos associados com a WTC, o NPS - Net Promoter Score e o CR - Chatbot Rates.

Estabelecido o modelo de avaliação, foi elaborado um experimento que incluía o convite aos alunos e a aceitação de um termo de consentimento livre e esclarecido, a aplicação de um questionário, o uso do Complex World, e a tabulação dos resultados.

$\mathrm{O}$ questionário implementado foi dividido em duas partes. Na parte inicial era realizado um pré-teste de conhecimento da língua, perguntas para estabelecer o perfil do estudante. Logo após essas atividades, os alunos recebiam as instruções para a utilização do jogo e eram direcionados ao jogo. Terminando de jogar, os alunos eram redirecionados para a parte final do questionário composta de um pós-teste e as perguntas baseadas no modelo de avaliação escolhido.

Para implementar os questionários foi utilizado o Google Forms para a construção e distribuição. Destacando que devido ao distanciamento social imposto pela pandemia de COVID-19, o experimento foi realizado de maneira digital e remota. 
Os itens avaliados foram: a motivação, a experiência do usuário com o jogo, a interlocução e questões de aprendizagem. Sobre cada item foram avaliadas as dimensões de motivação que compreende atenção, relevância, confiança e satisfação; a experiência do usuário que compreende a imersão, desafio, divertimento, competência e controle; a interlocução; a aprendizagem que inclui a aprendizagem de curto e longo termo.

Nas questões foi utilizada a técnica de Likert com um conjunto de respostas variando em uma escala de 1 ("discordo fortemente") a 5 ("concordo fortemente"). Complementarmente foram adicionadas questões discursivas para cada uma das dimensões para que o usuário pudesse se expressar de maneira livre sobre elas. Esta foi uma solução para tentar contornar o problema da falta de observação direta na aplicação do experimento e para ter respostas mais abrangentes conforme os princípios do Pensamento Complexo.

Para participar do experimento foram convidados alunos do ensino médio e do ensino superior. Os alunos receberam um $\operatorname{link} k^{2}$ para o questionário com as orientações iniciais. O período de aplicação foi de 01/02/2021 até 07/03/2021. Após o período de aplicação, iniciou-se a etapa de verificação dos resultados. Para isso utilizou-se uma planilha preparada com antecedência para receber os dados oriundos das respostas dos questionários.

\section{Resultados}

Nesta seção serão apresentados os resultados obtidos no experimento. O questionário inicial foi respondido por 143 estudantes.

Sobre o perfil dos usuários: $78 \%$ eram do sexo masculino e $22 \%$ do sexo feminino. A idade variou entre 14 e 58 anos, vale destacar que a maioria dos participantes estavam na faixa etária entre 14 e 20 anos (52,45\%). A maioria dos participantes foram do curso de Análise e Desenvolvimento de Sistemas (33,56\%) e do Bacharelado em Ciência da Computação (35,66\%). Estudantes do ensino médio formam 11,18\% do total.

Sobre a motivação foi possível verificar que a maioria dos estudantes concordaram que o jogo conseguiu manter a atenção e que o conteúdo do protótipo é relevante. Sobre a confiança, a maioria dos estudantes concordaram que o jogo proporciona a sensação de progresso. Também se verificou que os estudantes concordaram que o jogo aborda conteúdo importante e que conseguiram aplicar o que aprenderam nele. A Tabela 1 apresenta as repostas obtidas.

Tabela 1 - Dados sobre motivação

\begin{tabular}{|l|l|c|c|c|c|c|}
\hline \multirow{2}{*}{ Questão } & \multicolumn{5}{c|}{ Respostas (\%) } \\
\cline { 3 - 7 } & \multicolumn{1}{|c|}{$\mathbf{1}$} & $\mathbf{2}$ & $\mathbf{3}$ & $\mathbf{4}$ & $\mathbf{5}$ \\
\hline $\mathbf{1}$ & O jogo é atraente. & 0,00 & 7,02 & 22,81 & 22,81 & 47,37 \\
\hline $\mathbf{2}$ & $\begin{array}{l}\text { Houve algo interessante desde o início que capturou } \\
\text { minha atenção. }\end{array}$ & 3,51 & 3,51 & 26,32 & 26,32 & 40,35 \\
\hline $\mathbf{3}$ & $\begin{array}{l}\text { A variação dos diálogos ajudou a me manter atento ao } \\
\text { jogo. }\end{array}$ & 0,00 & 10,53 & 17,54 & 28,07 & 43,86 \\
\hline $\mathbf{4}$ & O conteúdo é relevante para os meus interesses. & 7,02 & 7,02 & 17,54 & 33,33 & 35,09 \\
\hline
\end{tabular}

2 Arquivo com as perguntas utilizadas: https://drive.google.com/file/d/1G1z9L462wa fVdcNLR1w0Vla_VyS1_2/view?usp=sharing https://drive.google.com/file/d/1TrPRqDxigDTPpZTb53XDDGewFX6-kuz0/view?usp=sharing 


\begin{tabular}{|c|c|c|c|c|c|c|}
\hline 5 & $\begin{array}{l}\text { O funcionamento é adequado ao meu jeito de } \\
\text { aprender. }\end{array}$ & 1,75 & 3,51 & 15,79 & 33,33 & 45,61 \\
\hline 6 & $\begin{array}{l}\text { O conteúdo está conectado com outros conhecimentos } \\
\text { que eu já possuía. }\end{array}$ & 0,00 & 3,51 & 15,79 & 17,54 & 63,16 \\
\hline 7 & $\begin{array}{l}\text { Foi fácil entender a forma de diálogo e começar a } \\
\text { utilizá-lo como material de estudo. }\end{array}$ & 0,00 & 3,51 & 8,77 & 24,56 & 63,16 \\
\hline 8 & $\begin{array}{l}\text { Estou satisfeito porque sei que terei oportunidades de } \\
\text { utilizar na prática coisas que aprendi. }\end{array}$ & 0,00 & 8,77 & 15,79 & 17,54 & 57,89 \\
\hline 9 & $\begin{array}{l}\text { É por causa do meu esforço pessoal que consigo } \\
\text { avançar e continuar progredindo }\end{array}$ & 0,00 & 3,51 & 24,56 & 14,04 & 57,89 \\
\hline
\end{tabular}

Sobre a experiência do usuário pode-se afirmar que a maioria dos estudantes concordam que existiu envolvimento com o jogo. Ainda é possível concluir que os usuários acharam o nível dos desafios compatíveis com o nível de habilidade. Os usuários também se sentiram satisfeitos com a experiência fornecida pelo jogo, bem como acreditam ter melhorado suas habilidades durante o jogo. As respostas obtidas são apresentadas na Tabela 2 .

Tabela 2 - Dados sobre a experiência do usuário

\begin{tabular}{|c|l|c|c|c|c|c|}
\hline \multirow{2}{*}{$\#$} & \multicolumn{1}{|c|}{ Questões } & \multicolumn{4}{|c|}{ Respostas (\%) } \\
\cline { 3 - 6 } & \multicolumn{1}{|c|}{$\mathbf{1}$} & $\mathbf{2}$ & $\mathbf{3}$ & $\mathbf{4}$ & $\mathbf{5}$ \\
\hline 1 & $\begin{array}{l}\text { Temporariamente esqueci as minhas preocupações do dia a dia, } \\
\text { fiquei totalmente concentrado no jogo. }\end{array}$ & 5,26 & 8,77 & 22,81 & 28,07 & 35,09 \\
\hline 2 & $\begin{array}{l}\text { Eu não percebi o tempo passar enquanto jogava, quando vi o } \\
\text { jogo acabou. }\end{array}$ & 5,26 & 10,53 & 21,05 & 22,81 & 40,35 \\
\hline 3 & $\begin{array}{l}\text { Me senti mais no ambiente do jogo do que no mundo real, } \\
\text { esquecendo do que estava ao meu redor. }\end{array}$ & 8,77 & 14,04 & 21,05 & 24,56 & 31,58 \\
\hline 4 & $\begin{array}{l}\text { Este jogo é adequadamente desafiador para mim, as tarefas não } \\
\text { são muito fáceis nem muito difíceis. }\end{array}$ & 8,77 & 10,53 & 31,58 & 19,30 & 29,82 \\
\hline 5 & $\begin{array}{l}\text { O jogo evolui num ritmo adequado e não fica monótono - oferece } \\
\text { novos obstáculos, situações ou variações de atividades. }\end{array}$ & 0,00 & 5,26 & 33,33 & 19,30 & 42,11 \\
\hline 6 & Me diverti com o jogo. & 1,75 & 1,75 & 21,05 & 14,04 & 61,40 \\
\hline 7 & $\begin{array}{l}\text { Quando interrompido, fiquei desapontado que o jogo tinha } \\
\text { acabado. }\end{array}$ & 5,26 & 12,28 & 28,07 & 15,79 & 38,60 \\
\hline 8 & Eu recomendaria este jogo para meus colegas. & 0,00 & 5,26 & 21,05 & 15,79 & 57,89 \\
\hline 9 & Gostaria de utilizar este jogo novamente. & 8,77 & 10,53 & 17,54 & 7,02 & 56,14 \\
\hline 10 & $\begin{array}{l}\text { Consegui atingir os objetivos do jogo por meio das minhas } \\
\text { habilidades. }\end{array}$ & 0,00 & 7,02 & 17,54 & 24,56 & 50,88 \\
\hline 11 & Tive sentimentos positivos de eficiência no desenrolar do jogo. & 1,75 & 8,77 & 12,28 & 24,56 & 52,63 \\
\hline 12 & Os controles para realizar ações no jogo responderam bem. & 1,75 & 5,26 & 12,28 & 14,04 & 66,67 \\
\hline 13 & É fácil aprender a usar a interface e controles do jogo. & 0,00 & 3,51 & 7,02 & 8,77 & 80,70 \\
\hline
\end{tabular}

Sobre a interlocução, o teste NPS, que versa sobre a receptividade do chatbot, mostrou que $49,12 \%$ dos usuários podem ser classificados como promotores, pois, segundo o teste, são os usuários que voltariam a utilizar o chatbot, 36,84\% podem ser classificados como neutros e são aqueles que tiveram uma experiência neutra, nem se entusiasmaram nem depreciaram o chatbot. Os classificados como detratores representam $14,03 \%$ dos usuários e são aqueles que não voltariam a utilizar o chatbot. O teste CR que verifica a utilidade das respostas fornecidas mostrou que $57,89 \%$ dos usuários concordam fortemente e $28,07 \%$ dos usuários concordam que as respostas foram úteis.

Com relação a vontade de se comunicar, na questão da autoconfiança, baseandose nas respostas dos estudantes, é possível afirmar que se sentiram confiantes para conversar com o chatbot. Quando perguntados sobre a percepção da autoconfiança, $68,42 \%$ responderam que se sentiram muito confiantes e outros $31,58 \%$ se sentiram confiantes no diálogo. Sobre o nervosismo da situação, $68,42 \%$ dos estudantes responderam que "não ficaria nervoso", 19,30\% que "eu ficaria um pouco nervoso", $8,77 \%$ que "eu ficaria nervoso" e 3,51\% que "definitivamente ficaria nervoso". E sobre 
se desejariam conversar novamente com o chatbot, 57,89\% responderam que sim e $35,09 \%$ de estudantes ficariam em dúvida de utilizar novamente. Pode-se afirmar, em geral, que o chatbot deixou os alunos mais confiantes para o diálogo e diminui o nervosismo da situação.

$\mathrm{Na}$ questão de aprendizagem foi possível comparar as respostas obtidas no préteste e no pós-teste conforme apresentado na Tabela 3. Para comparar os resultados obtidos foi realizado um teste-T de Student para verificar se existe uma diferença significativa entre as respostas da amostra no pré e no pós-teste. $\mathrm{O} p$-valor obtido é de $2,86 \times 10^{-2}$, sendo este valor menor que o $t$-crítico (alpha) de 0,05 . Pode-se afirmar, com $95 \%$ de confiança, que a hipótese nula é rejeitada. Ou seja, existe uma diferença significativa na média de acertos dos estudantes no pós-teste, indicando que o número de acertos depois de utilizar o protótipo foi significativamente maior do que no pré-teste. Conclui-se que o jogo se mostrou eficaz para os que participaram do experimento no concernente ao aprendizado.

Tabela 3 - Dados do pré e do pós teste

\begin{tabular}{|c|c|c|c|}
\hline & Pré $\%$ & Pós $\%$ & Diferença $\%$ \\
\hline Q1 & 98,11 & 98,11 & 0,00 \\
\hline Q2 & 100,00 & 100,00 & 0,00 \\
\hline Q3 & 96,23 & 98,11 & 1,89 \\
\hline Q4 & 84,91 & 94,34 & 9,43 \\
\hline Q5 & 100,00 & 100,00 & 0,00 \\
\hline Q6 & 92,45 & 94,34 & 1,89 \\
\hline Q7 & 100,00 & 100,00 & 0,00 \\
\hline Q8 & 100,00 & 100,00 & 0,00 \\
\hline Q9 & 98,11 & 98,11 & 0,00 \\
\hline Q10 & 88,68 & 98,11 & 9,43 \\
\hline Q11 & 94,34 & 98,11 & 3,77 \\
\hline Q12 & 100,00 & 100,00 & 0,00 \\
\hline Q13 & 92,45 & 98,11 & 5,66 \\
\hline Q14 & 100,00 & 100,00 & 0,00 \\
\hline
\end{tabular}

\section{Considerações Finais}

O Complex World apresentado mostrou-se um jogo capaz de auxiliar o aluno no aprendizado de inglês como segunda língua. $\mathrm{O}$ experimento realizado apresentou resultados positivos. Salienta-se também que devido às restrições impostas pela pandemia do novo coronavírus, não foi possível realizar a observação direta dos alunos interagindo com o jogo. O que deixa uma lacuna na análise da sua utilização. A estratégia para tentar contornar parcialmente o problema foi incentivar os alunos a expressar suas percepções nas questões discursivas.

Pode-se apontar como limitação deste trabalho, a avaliação de um grupo relativamente homogêneo formado principalmente por estudantes da área da computação que entendem a mecânica de um jogo e possuem, na maioria, facilidade para jogar e habilidade para utilizar o navegador e os recursos tecnológicos. Na tentativa de obter mais respostas de outras áreas foram enviados convites para estudantes da área de letras e de ciências humanas para participar do experimento, mas não foram aceitos.

Como trabalhos futuros podem ser inseridos novos conteúdos no protótipo e realizar avaliações em sala de aula comparando o ganho de conhecimento utilizando o jogo com o ensino tradicional. Outra melhoria pode ser a elaboração de modelos de avaliação para chatbots educacionais levando em consideração os conceitos presentes no Pensamento Complexo. 


\section{Referências}

Atwell, E. (1999). The language machine. London: The British Council.

Ayedoun, E., Hayashi, Y. e Seta, K. (2015). A Conversational Agent to Encourage Willingness to Communicate in the Context of English as a Foreign Language. Procedia Computer Science, v. 60, p. 1433-1442.

Behúnová, A., Ádám, N. e Pietriková, E. (nov 2016). Mobile support for learning of foreign languages. In 2016 International Conference on Emerging eLearning Technologies and Applications (ICETA).

Berns, A., Isla-Montes, J. L., Palomo-Duarte, M. e Dodero, J. M. (dez 2016). Motivation, students' needs and learning outcomes: a hybrid game-based app for enhanced language learning. SpringerPlus, v. 5, n. 1, p. 1305.

Busarello, R. I. (2016). Gamification: princípios e estratégias.

Collantes, X. R. (2013). Juegos y videojuegos. Formas de vivencias narrativas. Homo Videoludens 2.0. De Pacman a la gamification. Universidad Mayor; Universitat de Barcelona. .

De Gasperis, G. e Florio, N. (2012). Learning to Read/Type a Second Language in a Chatbot Enhanced Environment. Advances in Intelligent and Soft Computing. Berlin, Heidelberg: Springer Berlin Heidelberg. v. 152 AISCp. 47-56.

Do Amaral, L. A. M. (2007). Designing intelligent language tutoring systems for integration into foreign language instruction. ProQuest Dissertations and Theses, p. 197.

Edge, D., Searle, E., Chiu, K., Zhao, J. e Landay, J. A. (2011). MicroMandarin: Mobile language learning in context. In Conference on Human Factors in Computing Systems Proceedings. , CHI '11. Association for Computing Machinery. https://doi.org/10.1145/1978942.1979413.

Freire, M. M. (2013). Complex educational design: A course design model based on complexity. Campus-Wide Information Systems, v. 30, n. 3, p. 174-185.

Fryer, L. K., Nakao, K. e Thompson, A. (abr 2019). Chatbot learning partners: Connecting learning experiences, interest and competence. Computers in Human Behavior, v. 93, p. 279-289.

Furió, D., González-Gancedo, S., Juan, M. C., Seguí, I. e Costa, M. (2013). The effects of the size and weight of a mobile device on an educational game. Computers and Education, v. 64, n. May 2013, p. 24-41.

Hao, Y., Lee, K. S., Chen, S. T. e Sim, S. C. (2019). An evaluative study of a mobile application for middle school students struggling with English vocabulary learning. Computers in Human Behavior, v. 95, p. 208-216.

Ibáñez, M. B., García, J. J., Galán, S., Maroto, D. e Morillo, D. (2016). International Forum of Educational Technology \& Society Design and Implementation of a 3D MultiUser Virtual World for Language Learning Authors ( s ): María Blanca Ibáñez, José Jesús García, Sergio Galán, David Maroto, Diego Published by : Internatio. v. 14, n. 4.

Jing, X. (2017). Application of Mobile Learning System in Phonetics Teaching. In Proceedings of the 8th International Conference on E-Education, E-Business, EManagement and E-Learning - IC4E '17. , IC4E '17. ACM Press. 
https://doi.org/10.1145/3026480.3026486.

Kalita, O., Balykxina, T. e Pavlidis, G. (2015). Mobile navigator for learning foreign languages. In 2015 6th International Conference on Information, Intelligence, Systems and Applications (IISA).

Leffa, V. J. (2016). ReVEL na Escola: Ensinando a língua como um sistema adaptativo complexo. ReVEL, v. 14, p. 1-12.

MacIntyre, P. D., Dörnyei, Z., Clément, R. e Noels, K. A. (1998). Conceptualizing willingness to a L2: Communicate in confidence model situational. The Modern Language Journal, v. 82, n. 4, p. 545-562.

Morin, E. (2015). Introdução ao Pensamento Complexo. Porto Alegre: Editora Sulina.

Prensky, M. (2018). Digital Natives, Digital Immigrants. New Media and Society, v. 20, n. 11, p. 3961-3979.

Roggers, S. (2010). Level Up! The Guide to Great Video Game Design. John Wiley \& Sons.

Ruan, S., Willis, A., Xu, Q., et al. (2019). BookBuddy. In Proceedings of the Sixth (2019) ACM Conference on Learning@ Scale - L@S '19., L@S '19. ACM Press. https://doi.org/10.1145/3330430.3333643.

Savi, R., Wangenheim, C. G. Von, Ulbricht, V. R. e Vanzin, T. (2010). Proposta de um Modelo de Avaliação de Jogos Educacionais Introdução O Modelo de avaliação de treinamentos de Kirkpatrick. Novas Tecnologias na Educação, v. 8, n. 3, p. 12.

Silva, M. e Teles, V. D. (2011). O aprendizado de língua inglesa em dispositivos móveis através da aplicação de um sistema tutor inteligente. Anais do Simpósio Brasileiro de Informática na Educação, p. 542-545.

Steiner, K. E. e Tomkins, J. (2010). Narrative event adaptation in virtual environments.

Taalas, P., Jalkanen, J., Bradley, L., et al. (2018). Improve the chatbot performance for the DB-CALL system using a hybrid method and a domain corpus. Future-proof CALL: language learning as exploration and encounters - short papers from EUROCALL 2018, v. 2018, n. 2018, p. 100-105. 